\title{
Manipulation of Plasmonic Resonances in Graphene Coated Dielectric Cylinders
}

\author{
Lixin $\mathrm{Ge}^{1}$, Dezhuan $\mathrm{Han}^{1}$, and Ying $\mathrm{Wu}^{2}$ \\ ${ }^{1}$ Department of Applied Physics, Chongqing University, Chongqing 401331, China \\ ${ }^{2}$ Division of Computer, Electrical and Mathematical Sciences and Engineering \\ King Abdullah University of Science and Technology (KAUST), Thuwal 23955-6900, Saudi Arabia
}

\begin{abstract}
Graphene sheets can support surface plasmon as the Dirac electrons oscillate collectively with electromagnetic waves. Compared with the surface plasmon in conventional metal (e.g., $\mathrm{Ag}$ and $\mathrm{Au}$ ), graphene plasmonic owns many remarkable merits especially in Terahertz and far infrared frequencies, such as deep sub-wavelength, low loss, and high tunability. For graphene coated dielectric nano-scatters, localized surface plasmon (LSP)exist and can be excited under specific conditions. The LSPs are associated with the Mie resonance modes, leading to extraordinary large scattering and absorption cross section. In this work, we study systematically the optical scattering properties for graphene coated dielectric cylinders. It is found that the LSP can be manipulated by geometrical parameters and external electric gating. Generally, the resonance frequencies for different resonance modes are not the same. However, under proper design, we show that different resonance modes (e.g., dipole mode, quadruple mode etc.) can be excited at the same frequency. Thus, the scattering and absorption by graphene coated dielectric cylinders can indeed overcome the single channel limit. Our finding may open up new avenues in applications for the graphene-based $\mathrm{THz}$ optoelectronic devices.
\end{abstract}

